\title{
Radiometric Identification Based on Global Graph Fisher Analysis
}

\author{
Yongqiang $\mathrm{Jia}^{1,2}$ and $\mathrm{Lu} \mathrm{Gan}^{1}$ \\ ${ }^{1}$ Center for Cyber Security, School of Electronic Engineering, University of \\ Electronic Science and Technology of China, Chengdu, 611731, China \\ ${ }^{2}$ Southwest Electronics and Telecommunication Technology Research Institute, \\ Chengdu, 611731, China \\ jiayq0316@126.com
}

\begin{abstract}
In this paper, we propose a novel fingerprint learning algorithm for radiometric identification. There are many methods for fingerprint feature extraction which are based on the estimation of parameters of some post-modulator electronics, such as mixers, filters or amplifiers. However, the minute differences of different radio emitters are resulted from synthesis of many post-modulator electronics. The radio fingerprint features extracted with the proposed algorithm are discriminative representation of all the factors which contribute to the minuteness differences between different emitters. The proposed algorithm is based on characterizing the intraclass compactness and the interclass separability by preserving both local manifold structure and the global discriminant information of signal data set. The interclass separability is modeled by a penalty graph characterizing the distances between all interclass samples, where the intraclass compactness is modeled by an intrinsic graph characterizing the relationships of all intraclass samples. Since both class label information and graph embedding structure are considered, the proposed algorithm can obtain better performance in radiometric identification applications. Experiments on real data sets show the effectiveness of the proposed algorithm for tasks of radio emitter identification. The results indicate that the proposed algorithm can discriminate the emitters of inter-manufacturer with an identification accuracy of $96 \%$, and can discriminate the emitters of intra-manufacturer with the best classification accuracy over $99 \%$ and the worst classification accuracy of $78 \%$.
\end{abstract}

Keywords: Radio Emitter Identification, Fingerprint Learning, Marginal Fisher Analysis

\section{Introduction}

The security of wireless communication system has been widely studied in recent years. Since the intruder may duplicate the secure tokens or passwords, the inherent physical layer characteristics of the radio emitters have been exploited to improve the security mechanism [1-2]. There are minute differences between different radio emitters because of imperfect manufacturing processes. Therefore, it is possible to identify different radio emitters by extracting a unique radio fingerprint from the received signals. Radiometric identification technique has been reported to extract the radio frequency (RF) fingerprint features from signals. The extracted RF fingerprint features can be used to support the applications of wireless communication security, intrusion detection and authentication [3-4].

The signals emitted from radio emitters not only include the modulated signal section but also include the unintended modulation section which results from many idiosyncratic 
hardware properties. The modulated signal section is the period of data transmission such as preamble and payload, while the unintended modulation section is not related to data transmission. In most cases, unintentional emissions can be observed in the duration of transient signal because the power level usually increases gradually during the period of emitter power-on. The amplitude and phase profiles of transient signal are different between different radio emitters because of the idiosyncratic characteristics of power amplifiers, filters and frequency synthesizers. It has been shown that the RF fingerprint features extracted from transient signals for radiometric identification can obtain good classification performance [5-6]. The accuracy of the detection and separation of the turnon transients is crucial to the overall identification performance. The Bayesian ramp detector, variance trajectory detector and correlation detector have been reported for the detection of the start instants of transients [7-9]. The RF fingerprint features are generated from instantaneous amplitude, phase, and frequency responses of transient signal [10]. Extraction of the features can be conducted in time domain, spectral domain, and transform domain [11]. However, the discriminatory performance of transient analysis is not always good when the emitters are the same model [12-13]. The underlying reason is that the detection of the exact turn-on time instant of transient is difficult because of the unstable profiles of transient [9]. Another reason which makes transient analysis impractical may be that it requires high-end receiver to offer high oversampling rate [14]. The work presented by Danev and Capkun has shown that low equal error rates (EER) can be achieved with a high-end receiver [15].

The steady-state signal is defined as the signal section truncated from the end of the transient signal forwards for duration of several symbols. The methods that extract RF fingerprint features from steady-state signal are also called as modulation-based approaches [16-17]. In contrast with the case of transient analyses, the modulation-based approaches just require low-end receiver. Recently, Brik et al. have developed a method called Passive Radiometric Device Identification System (PARADIS) to extract the RF fingerprints from steady-state signal [18]. The PARADIS method extracts six fingerprint features from IEEE 802.11 frames to discriminate 138 emitters with a high accuracy. However, the signals are collected with a vector signal analyzer at distances of 15 meters from the antenna in their experiments. In another recent study, Scanlon et al. have applied the spectral averaging technique and feature subset selection technique to achieve the identification accuracy of $99.8 \%$ [16]. However, the method in [16] is evaluated under the condition that the transmitters and the receivers are located in an anechoic chamber. In fact, the receiver is usually as far as several kilometers to the transmitter in practice. In steady-state analyses, the RF fingerprint features are usually extracted from preamble section used for synchronization and Doppler offset removal in communication system.

In contrast with the work of transient analysis and modulation-based approaches, Polak et al. have exploited the nonlinearities of power amplifier (PA) and digital-to-analog converter (DAC) to uniquely identify wireless emitters [21]. The nonlinearity of PA is represented with volterra series and the integral nonlinearity (INL) of the DAC is modeled with a Brownian Bridge random process.

In most of the cases, it is necessary to reduce the amount of features. Dimensionality reduction technique is used to find the discriminant subspace by selecting the relevant information and reducing the redundant information. The extracted RF fingerprint features with many elements can be compressed to a low dimension vector containing the discriminant information. Recently, Padilla et al. have presented an identification method based on RF fingerprint feature reduction [22]. Their experimentations on Wi-Fi signals show the effectiveness of the identification algorithm using the reduction technique of Partial Least Squares regression (PLS). Principal Component Analysis (PCA) is another technique used to reduce the dimensionality of RF fingerprint features [23]. Besides, Linear Discriminant Analysis (LDA) can preserve discriminative information between different data sets, and Marginal Fisher Analysis (MFA) can discriminate different classes 
by using the local manifold structure [24-25]. However, MFA improves the classification performance little because it doesn't consider the distances between the points which are not in a neighborhood.

Unlike the previous state-of-art techniques, the RF fingerprint features extracted in this paper aren't based on the estimation of physical parameters. Published works usually concern on the features derived from some of post-modulator electronics (mixers, filters or amplifiers), while the minute differences of different radio emitters are resulted from synthesis of many post-modulator electronics. Inspired by this, we propose a radiometric identification technique extracting all discriminative features from regions of interest (ROI) signals. The proposed radiometric identification algorithm based on Global Graph Fisher Analysis (GGFA) considers the intraclass compactness and the interclass separability, simultaneously. The former is modeled by an intrinsic graph characterizing the relationships of all intraclass samples, while the latter is modeled by a penalty graph characterizing the distances between all inter-class samples. Since both class label information and graph embedding structure are considered, GGFA can obtain better performance in radiometric identification applications.

The rest of this paper is organized as follows: Section II provides a background of radio fingerprint identification; Section III presents the proposed algorithm; Section IV analyzes the classification results; and Section V concludes the paper.

\section{Radio Fingerprint Identification}

Usually, wireless communication security systems use the radiometric identification technique to solve a close-set identification problem. A training procedure is conducted by the security system to collect signals and extract RF features from the legitimate emitters. Then, the RF features from an unknown emitter is extracted and compared with the stored features of legitimate emitters in the stage of testing. There are various algorithms used to classify the label of the unknown emitter. In the application of intrusion detection, the unknown emitter may be illegal and then it is an open-set identification problem. Radiometric identification consists of the steps of signal collection, data preprocessing, feature extraction and classification. The procedure of radiometric identification is shown in Figure 1.

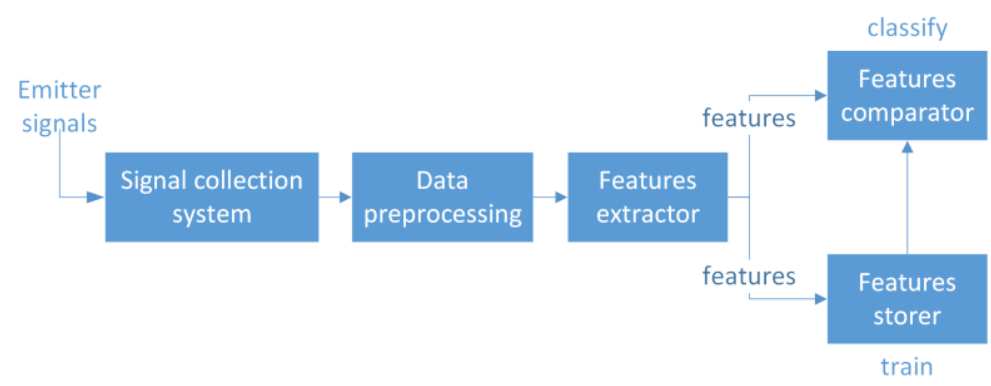

Figure 1. Procedure of Radiometric Identification

\subsection{Signal Collection \& Detection}

Firstly, the signals are collected with a signal collection system. The RF range of this system is $20.0 \mathrm{MHz}$ to $6000.0 \mathrm{MHz}$. The interest signal band is down-converted and digitized with a 12-bit analog-to-digital converter. The data should be preprocessed by Hilbert transform to obtain the complex-valued data format. During the period of data preprocessing, the exact turn-on time instant of radio emitter can be detected with Bayesian ramp detector or variance trajectory detector [7-8]. The transient section and steady-state signal section can be truncated as ROI signals. 


\subsection{Feature Extraction}

After the steps of signal collection and data preprocessing, RF fingerprint features can be extracted from ROI signals. Figure 2 shows the normalized amplitude of two bursts extracted from an emitter. There are lists of RF fingerprint features in the literatures. PCA, LDA and PLS can be used to reduce the dimensionality of the extracted RF fingerprint features [22-23]. In contrast with the published works, the low-dimensional RF fingerprint features are learned directly in this paper. A feature extractor which can directly extract effective and discriminative features from signal data is trained in the stage of training. In the stage of testing, the trained extractor is used to extract the RF fingerprint features from signals of an unknown emitter.

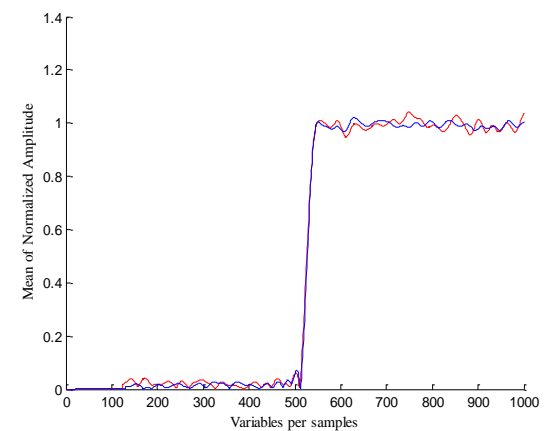

Figure 2. Normalized Amplitude Extracted from an Emitter (Two Burst Data)

\subsection{Classification}

There are many classifiers used to compare the stored features of training set and the features of an unknown emitter in the open literature. The performance of the classifier is usually depended on the features and the applications. It can be a simply measure of the Euclidean distance between the features or a complex machine learning algorithm. In [20], it compares the impact of $k$-nearest neighbours (KNN) and support vector machine (SVM) on the classification of different Wi-Fi devices. The results of experimentations show that it can achieve lower identification error rate by using SVM classifier. Besides, in [8] and [21], probabilistic neural networks (PNN) and generalized likelihood ratio test (GLRT) are also used to obtain good performance for radiometric identification, respectively.

\section{Proposed Method}

\subsection{Problem Statement}

In this paper, the transient section and preamble section are truncated as ROI signals. The RF fingerprint features are extracted from the ROI signals because they theoretically should be the same for all emitters. Practically, there are minute differences in the transient section and preamble section because of imperfect manufacturing processes and tolerances design of analog components. Usually, the ROI signal is a vector with a high dimensionality. It should be compressed to a low dimensional vector, which can represent the discriminant information effectively.

Assume we have a training data set $\mathbf{X}=\left\{\mathbf{X}_{1}, \cdots, \mathbf{X}_{C}\right\}, \mathbf{X}_{i}=\left[x_{i l}, \cdots, x_{i n}\right](1 \leq i \leq C), C$ is the number of emitter class and $n$ is the number of training samples from each class, $x_{i j}$ is the ${ }^{j \text { th }}$ training sample from class $i, x_{i j} \in \mathfrak{R}^{M}(1 \leq i \leq C, 1 \leq j \leq n)$. Given a testing 
sample from an unknown emitter, we predict the class label of the emitter by comparing its features with the features of training data. The classifier used in this paper is the SVM classifier. Similar to dimensionality reduction, the training samples should be projected to a low-dimensional space to extract the discriminative features:

$$
y=\mathbf{W}^{T} x
$$

where the superscript $T$ denotes the transpose. ${ }^{x}$ is a training sample and ${ }^{y}$ is its feature vector. The dimension of $x$ is $M$, while the dimension of ${ }^{y}$ is $m, m \square M$. The feature extractor $\mathbf{W}$ should be trained during the training period with known training samples and associating class labels. There are many published works solving the supervised learning problem similar to (1) in feature selection and feature reduction.

\subsection{Previous Works}

In the graph embedding framework, a graph $G=\{\mathbf{X}, \mathbf{S}\}$ is constructed to represent the relationships of data in $\mathbf{X}$ [25]. Each data is mapped to a vertex in the graph. The relationships of all data pairs are characterized by a similarity matrix $\mathbf{S}$. The geometry structure of the data set in high-dimensional space should be preserved in a lowdimensional space by the projection matrix $\mathbf{W}$. The optimization problem for the projection matrix $\mathbf{W}$ is [24-25]:

$$
\mathbf{W}^{*}=\underset{\operatorname{Tr}\left(\mathbf{W}^{T} \mathbf{X D X} \mathbf{X}^{T} \mathbf{W}\right)=\mathbf{I}_{a}}{\operatorname{argmin}} \sum_{i \neq j}\left\|\mathbf{W}^{T} x_{i}-\mathbf{W}^{T} x_{j}\right\|^{2} S_{i j}
$$

After some algebraic formulations, it can be written as:

$$
\begin{gathered}
\mathbf{W}^{*}=\underset{\operatorname{Tr}\left(\mathbf{W}^{T} \mathbf{X D M} \mathbf{X}^{T} \mathbf{W}\right)=\mathbf{I}_{a}}{\operatorname{argmin}} \operatorname{Tr}\left(\mathbf{W}^{T} \mathbf{X L}\left(\mathbf{W}^{T} \mathbf{X}\right)^{T}\right) \\
\mathbf{L}=\mathbf{D}-\mathbf{S}
\end{gathered}
$$

where $\mathbf{I}_{a}$ is a unit matrix, $\mathbf{S}$ is the similarity matrix with elements of $S_{i j}, \mathbf{D}$ is a diagonal matrix with $D_{i i}=\sum_{j=1}^{n} S_{i j}$, and $\mathbf{L}$ is the corresponding Laplacian matrix.

The LDA algorithm and MFA algorithm can be reformulated in this framework with different graphs and embedding types [25].

\section{A. LDA}

LDA tries to find those vectors that discriminate different classes by preserving the global geometric structure. The intraclass scatter matrix $\mathbf{S}_{w}$ is minimized and the interclass scatter matrix $\mathbf{S}_{b}$ is maximized at the same time. The matrix $\mathbf{S}_{w}$ and $\mathbf{S}_{b}$ are given as [25]:

$$
\begin{aligned}
& S_{b}=\sum_{i=1}^{C} m_{i}\left(\mu_{i}-\mu_{0}\right)\left(\mu_{i}-\mu_{0}\right)^{T} \\
S_{w}= & \sum_{i=1}^{C}\left(\sum_{j=1}^{m_{i}}\left(x_{i j}-\mu_{i 0}\right)\left(x_{i j}-\mu_{i 0}\right)^{T}\right) \\
= & \mathbf{X}\left(\mathbf{I}-\sum_{i=1}^{C} \frac{1}{m_{i}} e_{i} e_{i}^{T}\right) \mathbf{X}^{T}
\end{aligned}
$$

where $C$ is the number of class, $x_{i j}$ is the ${ }^{j \text { th }}$ training sample in class ${ }^{i}, \mu_{0}$ is the total mean of all samples, $\mu_{i 0}$ is the mean of samples in class ${ }^{i}$. There are $\sum_{i=1}^{c} m_{i}$ zeros in $\mathbf{e}_{i}$ and $\mathbf{e}_{i}(j)=1$ if $j=i, m_{i}$ is the number of samples in class ${ }^{i}$. 
The goal is to maximize the ratio:

$$
\mathbf{W}_{L D A}=\arg \min _{\mathbf{W}} \frac{\operatorname{Tr}\left[\mathbf{W}^{T} S_{w} \mathbf{W}\right]}{\operatorname{Tr}\left[\mathbf{W}^{T} S_{b} \mathbf{W}\right]}
$$

The data should be of a Gaussian distribution in LDA. However, it is often not satisfied in real-world problems.

\section{B. MFA}

Based on the graph embedding framework, MFA applies a penalty graph to model the interclass separability and an intrinsic graph to model the intraclass compactness [24]. The intraclass scatter matrix $\mathbf{S}_{w-M F A}$ and the interclass scatter matrix $\mathbf{S}_{b-M F A}$ are given as [24]:

$$
\begin{gathered}
\mathbf{S}_{w-M F A}=\sum_{i} \sum_{i \in N_{k 1}(j)} \| \mathbf{W}^{T} x_{i}-\mathbf{W}_{k 1}(i) \\
= \\
=2 \operatorname{Tr}\left[\mathbf{W}^{T} \mathbf{X}_{j} \|^{2}\left(\mathbf{D}^{I}-\mathbf{S}^{I}\right) \mathbf{X}^{T} \mathbf{W}\right] \\
S_{i j}^{I}= \begin{cases}1, & \text { if } i \in N_{k 1}(j) \text { or } j \in N_{k 1}(i) \\
0, & \text { else }\end{cases} \\
\mathbf{S}_{b-M F A}=\sum_{(i, j) \in P_{k 2}\left(\pi_{i}\right)} \sum_{(i, j) \in P_{k 2}\left(\pi_{j}\right)}\left\|\mathbf{W}^{T} x_{i}-\mathbf{W}^{T} x_{j}\right\|^{2} \\
=2 \operatorname{Tr}\left[\mathbf{W}^{T} \mathbf{X}\left(\mathbf{D}^{P}-\mathbf{S}^{P}\right) \mathbf{X}^{T} \mathbf{W}\right] \\
S_{i j}^{P}= \begin{cases}1, & \text { if }(i, j) \in P_{k 2}\left(\pi_{i}\right) \text { or }(i, j) \in P_{k 2}\left(\pi_{j}\right) \\
0, & \text { else }\end{cases}
\end{gathered}
$$

where $N_{k 1}(i)$ is a set of the $k 1$ nearest neighbors of ${ }^{x_{i}}$, which are from the same class, $P_{k 2}\left(\pi_{i}\right)$ denotes a set of $k 2$ nearest data pairs $\left(x_{i}, x_{j}\right)$, which are from different classes, $\pi_{i}$ is the label of $x_{i} . \mathbf{S}^{I}$ and $\mathbf{S}^{P}$ are similarity matrix with elements of $S_{i j}^{I}$ and $S_{i j}^{P}$ respectively, $\mathbf{D}^{I}$ and $\mathbf{D}^{P}$ are corresponding diagonal matrix. The optimal projection matrix $\mathbf{W}_{M F A}$ can be computed as:

$$
\mathbf{W}_{M F A}=\arg \min _{\mathbf{W}} \frac{\operatorname{Tr}\left[\mathbf{W}^{T} \mathbf{X}\left(\mathbf{D}^{I}-\mathbf{S}^{I}\right) \mathbf{X}^{T} \mathbf{W}\right]}{\operatorname{Tr}\left[\mathbf{W}^{T} \mathbf{X}\left(\mathbf{D}^{P}-\mathbf{S}^{P}\right) \mathbf{X}^{T} \mathbf{W}\right]}
$$

\subsection{Proposed GGFA}

The penalty graph constructed in MFA only punishes the connections between marginal points which are $k$ nearest interclass pairs. However, it ignores the relationships of other vertex pairs even if they are from different classes. So the penalty graph in MFA can't ensure the separability of interclass vertex pairs which are not in a neighborhood. This will lead to a poor generalization performance in classification tasks. Meanwhile, the intrinsic graph in MFA also only concentrates on the connections of intraclass vertex pairs which are in a neighborhood. However, there is a lack of the information of other intraclass vertex pairs if they are not in a neighborhood. This also may deteriorate the performance of MFA.

Motivated by the graph embedding framework, we propose a supervised feature learning algorithm based on preserving the local manifold structure and the global information. The proposed algorithm constructs a new penalty graph characterizing the distances between all interclass vertex pairs, and constructs a new intrinsic graph characterizing the relationships of all intraclass vertex pairs. 
The original data samples can be mapped to a low-dimensional space by the projection matrix as in (1). The embedding of vertex $x_{i j}$ is the lower dimension vector $y_{i j}$, $y_{i j} \in \mathfrak{R}^{m}(1 \leq i \leq C, 1 \leq j \leq n), \quad m \square M$. The feature set is $\mathbf{Y}=\left\{\mathbf{Y}_{1}, \cdots, \mathbf{Y}_{C}\right\}$, where $\mathbf{Y}_{i}=\left[y_{i l}, \cdots, y_{i n}\right]$. For each data sample ${ }^{x_{i}}$, we find its nearest neighbors and let $N\left(x_{i}\right)$ be the set of its nearest neighbors. There are two subsets in $N\left(x_{i}\right), N_{b}\left(x_{i}\right)$ and $N_{w}\left(x_{i}\right)$. Let $l(x)$ be the class label of $x$. Specifically,

$$
\begin{aligned}
& N_{b}\left(x_{i}\right)=\left\{x_{j} \mid l\left(x_{j}\right) \neq l\left(x_{i}\right), x_{j} \in N\left(x_{i}\right)\right\} \\
& N_{w}\left(x_{i}\right)=\left\{x_{j} \mid l\left(x_{i}\right)=l\left(x_{j}\right), x_{j} \in N\left(x_{i}\right)\right\}
\end{aligned}
$$

We construct two graphs: intrinsic graph $G_{I}=\left\{\mathbf{X}, \mathrm{S}_{I}\right\}$ and penalty graph $G_{P}=\left\{\mathbf{X}, \mathrm{S}_{P}\right\}$. $\mathbf{X}$ is the training data set for both graphs. ${ }^{S_{I}}$ and $S_{P}$ are similarity matrixes of the intrinsic graph and the penalty graph, respectively. The elements of similarity matrix are distance measurements of vertex pairs in graphs.

For the penalty graph, we explore a new similarity matrix to characterize the interclass separability. Let $N_{\text {inter }}$ be the set of interclass vertex pairs. According to the distances between the vertexes, it consists of two subsets, $N_{\text {inter }-n}$ and $N_{\text {inter }-f}$. The interclass vertex pairs in set $N_{\text {inter-n }}$ are marginal points.

$$
\begin{gathered}
N_{\text {inter }-n}=\left\{\left(x_{i}, x_{j}\right) \mid \begin{array}{c}
x_{i} \in N_{b}\left(x_{j}\right) \\
\text { or } x_{j} \in N_{b}\left(x_{i}\right)
\end{array}\right\} \\
N_{\text {inter }-f}=\left\{\left(x_{i}, x_{j}\right) \mid \begin{array}{c}
l\left(x_{j}\right) \neq l\left(x_{i}\right) \\
\text { and } x_{i} \notin N_{b}\left(x_{j}\right) \\
\text { and } x_{j} \notin N_{b}\left(x_{i}\right)
\end{array}\right\}
\end{gathered}
$$

The optimal projection $\mathbf{W}$ should be computed as

$$
\begin{gathered}
\mathbf{W}=\arg \max _{\mathbf{W}} \sum_{p=1}^{c} \sum_{\substack{q=1 \\
q \neq p}}^{c} \sum_{i=1}^{n} \sum_{j=1}^{n}\left\|\mathbf{W}^{T} x_{p i}-\mathbf{W}^{T} x_{q j}\right\|^{2} S_{\mathrm{P}, i j} \\
=\arg \max _{\mathbf{W}} \mathbf{W}^{T} \mathbf{X}\left(\mathbf{D}_{P}-\mathbf{S}_{P}\right) \mathbf{X}^{T} \mathbf{W} \\
=\arg \max _{\mathbf{W}} \mathbf{W}^{T} \mathbf{X} \mathbf{L}_{P} \mathbf{X}^{T} \mathbf{W} \\
\mathbf{L}_{P}=\mathbf{D}_{P}-\mathbf{S}_{P} \\
\mathbf{D}_{P}=\sum_{j} S_{P, i j}
\end{gathered}
$$

where $\mathbf{L}_{P}$ is the Laplacian matrix of $G_{P}$, and $\mathbf{D}_{P}$ is the corresponding diagonal matrix. Defining:

$$
\begin{gathered}
S_{\mathrm{P}, i j}=\left\{\begin{array}{c}
\exp \left(-\operatorname{Dis}\left(x_{i}, x_{j}\right)\right),\left(x_{i}, x_{j}\right) \in N_{\text {inter }-n} \\
\exp \left(\operatorname{Dis}\left(x_{i}, x_{j}\right)\right),\left(x_{i}, x_{j}\right) \in N_{\text {inter }-f}
\end{array}\right. \\
\operatorname{Dis}\left(x_{i}, x_{j}\right)=\left\|x_{i}-x_{j}\right\|^{2}
\end{gathered}
$$

Since ${ }^{y=\mathbf{w}^{T} x}$, according to (17) and (20), the interclass marginal vertex pairs, i.e. $\left(\mathbf{x}_{i}, \mathbf{x}_{j}\right) \in N_{\text {inter }-n}$, should be mapped far apart. It means the closer these original marginal 
samples are, the farer the mapped points are. Meanwhile, the interclass vertex pairs in set $N_{\text {inter- } f}$ should also be mapped far apart. This is an important characteristic of the penalty graph, and it is different from that in MFA. It can be seen from (20) that the punishments in GGFA include both the relationships of marginal points in $N_{\text {inter } n}$ and the interclass vertex pairs in $N_{\text {inter }-f}$.

Similarly, for the intrinsic graph, the optimal projection $\mathbf{W}$ should be computed as:

$$
\begin{gathered}
\mathbf{W}=\arg \min _{\mathbf{W}} \sum_{p=1}^{C} \sum_{i=1}^{n} \sum_{\substack{j=1 \\
j \neq i}}^{n}\left\|\mathbf{W}^{T} x_{p i}-\mathbf{W}^{T} x_{p j}\right\|^{2} S_{I, i j} \\
=\arg \min _{\mathbf{W}}\left(\mathbf{W}^{T} \mathbf{X D}_{I} \mathbf{X}^{T} \mathbf{W}-\mathbf{W}^{T} \mathbf{X} \mathbf{S}_{I} \mathbf{X}^{T} \mathbf{W}\right) \\
\mathbf{D}_{I}=\sum_{j} \mathbf{S}_{I, i j}
\end{gathered}
$$

We put a constraint on the mapped points to avoid a degraded solution:

$$
y \mathbf{D}_{I} y^{T}=1
$$

So (22) is equivalent to maximize the following function:

$$
\begin{aligned}
& \mathbf{W}=\arg \max _{\mathbf{W}}\left(\mathbf{W}^{T} \mathbf{X} \mathbf{S}_{I} \mathbf{X}^{T} \mathbf{W}\right) \\
& \text { s.t. } \mathbf{W}^{T} \mathbf{X D}_{I} \mathbf{X}^{T} \mathbf{W}=\mathbf{I}
\end{aligned}
$$

We define:

$$
\mathbf{S}_{\mathrm{L}, i j}=\left\{\begin{array}{l}
\exp \left(-1 / \operatorname{Dis}\left(x_{i}, x_{j}\right)\right),\left(x_{i}, x_{j}\right) \in N_{\text {intra }-n} \\
\exp \left(-\operatorname{Dis}\left(x_{i}, x_{j}\right)\right),\left(x_{i}, x_{j}\right) \in N_{\text {intra }-f}
\end{array}\right.
$$

where $\operatorname{Dis}\left(x_{i}, x_{j}\right)$ is defined as in (21). Let $N_{\text {intra }}$ be the set of intraclass vertex pairs. This set can be divided into two subsets, $N_{\text {intra-n }}$ and $N_{\text {intra-f }}$.

$$
\begin{gathered}
N_{\text {intra-n }}=\left\{\left(x_{i}, x_{j}\right) \mid \begin{array}{c}
x_{i} \in N_{w}\left(x_{j}\right) \\
\text { or } x_{j} \in N_{w}\left(x_{i}\right)
\end{array}\right\} \\
N_{\text {intra-f }}=\left\{\left(x_{i}, x_{j}\right) \mid \begin{array}{c}
l\left(x_{j}\right)=l\left(x_{i}\right) \\
\text { and } x_{i} \notin N_{w}\left(x_{j}\right) \\
\text { and } x_{j} \notin N_{w}\left(x_{i}\right)
\end{array}\right\}
\end{gathered}
$$

It can be seen from (26) that we model the intraclass compactness with a new intrinsic graph which is different from that in MFA. In the proposed GGFA, the original intraclass vertex pairs in set $N_{\text {intra-n }}$ should be mapped close, and the intraclass vertex pairs in set $N_{\text {intra-f }}$ also should be mapped close.

GGFA not only considers the local manifold structure but also uses the global information. GGFA maximizes the interclass separability and minimizes the intraclass compactness at the same time. The combination of the optimal projection in (17) and (25) leads to:

$$
\begin{aligned}
& \mathbf{W}_{G G F A}=\arg \max _{\mathbf{W}}\left(\mathbf{W}^{T} \mathbf{X}\left(\beta \mathbf{L}_{P}+(1-\beta) \mathbf{S}_{I}\right) \mathbf{X}^{T} \mathbf{W}\right) \\
& \text { s.t. } \mathbf{W}^{T} \mathbf{X D}_{I} \mathbf{X}^{T} \mathbf{W}=\mathbf{I}
\end{aligned}
$$

By some algebraic formulations, it reduces to find the maximum eigenvalue solution to the generalized eigenvalue problem:

$$
\mathbf{X}\left(\beta \mathbf{L}_{P}+(1-\beta) \mathbf{S}_{I}\right) \mathbf{X}^{T} \mathbf{W}_{G G F A}=\lambda \mathbf{X} \mathbf{D}_{I} \mathbf{X}^{T} \mathbf{W}_{G G F A}
$$


$\beta(0 \leq \beta \leq 1)$ is a tradeoff parameter used for controlling the weight of interclass separability.

\subsection{Radiometric Identification with GGFA}

There are two stages for radiometric identification with the proposed GGFA. Firstly, in the stage of training, the signals of radio emitters should be collected and preprocessed, and the feature extractor should be trained using the training data set. The features of training samples can be extracted with the feature extractor and be stored. The SVM classifier should also be trained with these features. Then, in the stage of testing, the features of an unknown emitter can be extracted, and be compared with the stored features. The SVM classifier predicts the class label of the unknown emitter. The procedure of radiometric identification with GGFA is illustrated in Figure 3. The procedure can be implemented as follows:

\section{Algorithm: GGFA for radiometric identification Training Stage}

Step 1: Collect signals of the known emitters and truncate the transient section and preamble section as ROI signals;

Step 2: Preprocess the ROI signals and calculate the normalized instantaneous amplitude, instantaneous phase to construct the training set $\mathbf{X}$;

Step 3: Construct the penalty graph $G_{P}=\left\{\mathbf{X}, \mathrm{S}_{P}\right\}$ and intrinsic graph $G_{I}=\left\{\mathbf{X}, \mathrm{S}_{I}\right\}$, according to (20) and (26) respectively;

Step 4: Solve the generalized eigenvalue problem of (30) to train the feature extractor $\mathbf{W}$;

Step 5: Extract the features of all training samples with $\mathbf{W}$ using (1) and store these features;

Step 6: Train the SVM classifier with the stored features;

Testing Stage

Step 7: Collect signals of the unknown emitter and truncate the transient section and preamble section as ROI signal;

Step 8: Preprocess the ROI signal to get the testing sample ${ }^{x_{u}}$;

Step 9: Extract feature of the testing sample $y_{u}$ with the extractor $\mathbf{W}$ according to (1);

Step 10: Predict the class label of the unknown emitter with the trained SVM by comparing the distance of $y_{u}$ and the stored features. 


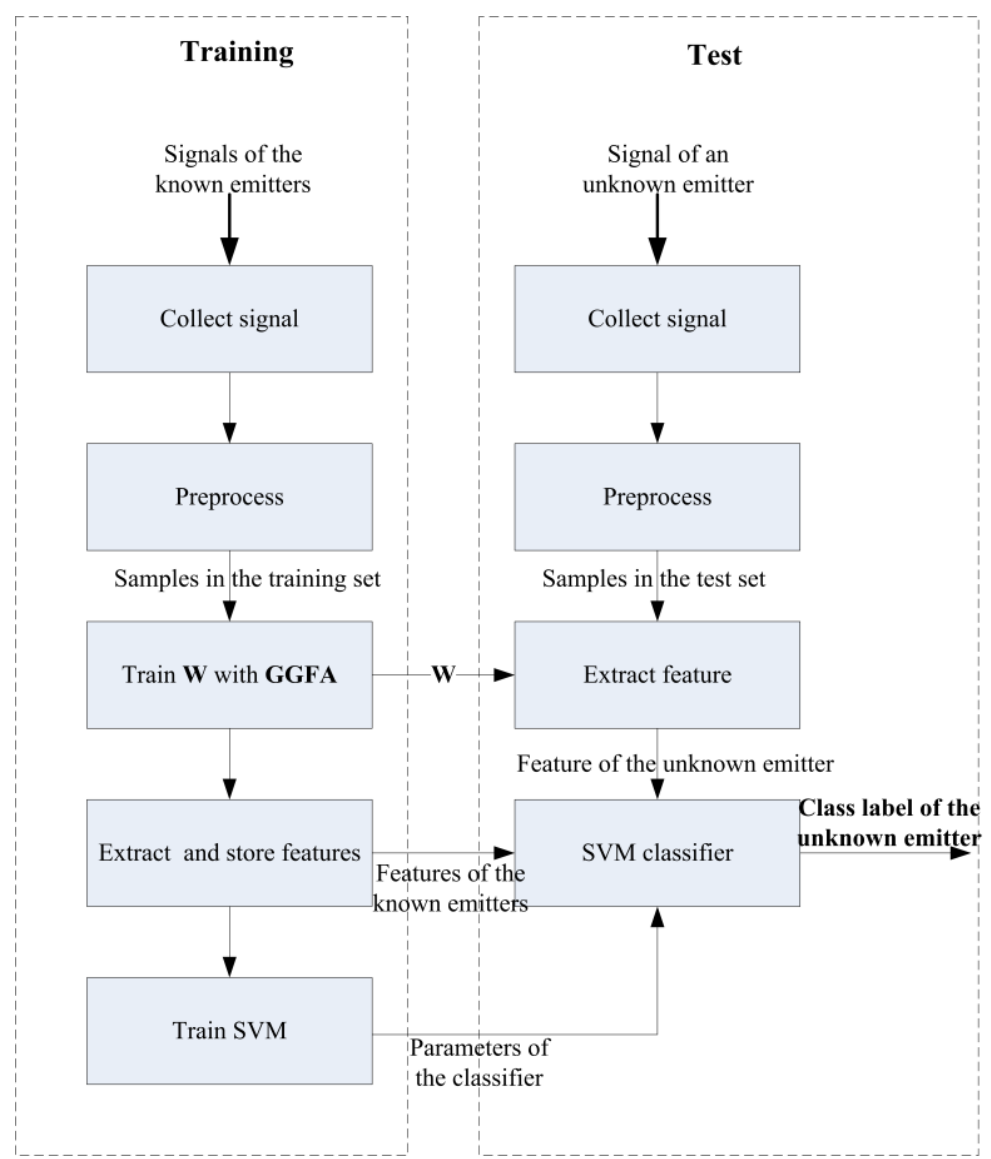

Figure 3. The Procedure of Radiometric Identification with GGFA

\section{Experiments Results}

\subsection{The Performance of Radiometric Identification with GGFA}

The performances of inter-manufacturer classification and intra-manufacturer classification are evaluated in this subsection. The evaluation is conducted with signals captured in the real-world environment.

\section{A. Evaluation setup}

The Automatic Identification System (AIS) signals emitted from ships are collected by an antenna located near a port in China. The center frequency of AIS burst signal is $161.975 \mathrm{MHz}$ or $162.025 \mathrm{MHz}$. The data bits are modulated with Gaussian Minimum Shift Keying (GMSK) of the bandwidth of $25 \mathrm{kHz}$, and the data rate is 9600 bits per second. Burst signals from six AIS emitters are measured by a signal analyzer to construct the data set. These six emitters are from three manufacturers because two emitters from each group have successive Maritime Mobile Service Identify (MMSI) numbers, and are located on sister ferry boats. The first emitter and the second emitter are from the same manufacturer. The third emitter and the fifth emitter are from another manufacturer. The fourth emitter and the sixth emitter are from the third manufacturer, as illustrated in Table 1. There are 140 data samples in each class, which result in a data set of 840 data samples. To conduct the experiment, we use the one-leave-out strategy to split the data set into training set and testing set. The signal-to-noise ratio (SNR) for all data samples is about $18 \mathrm{~dB}$. The learned feature extractor is used to extract the features of testing data samples, and predict their class label. The parameter $\beta$ is selected as 0.1 . 
Table 1. Emitters from 3 Manufacturers

\begin{tabular}{|c|c|c|c|c|c|c|}
\hline & Emitter 1 & Emitter2 & Emitter3 & Emitter4 & Emitter5 & Emitter6 \\
\hline Manufacturer 1 & + & + & & & & \\
\hline Manufacturer2 & & & + & & + & \\
\hline Manufacturer3 & & & & + & & + \\
\hline
\end{tabular}

\section{B. Features extracted with GGFA}

The features of these six AIS emitters are extracted from their ROI signals. Figure 4 gives the view of two features extracted with GGFA. The results show that the proposed GGFA is useful for identification of different emitters. It can be seen from Figure 4 that the features of six emitters which are from three manufacturers are separated into several blocks. This reveals that GGFA can be used to discriminate emitters from different manufacturers. It can also be observed that there may be overlaps of features between different emitters. The reason is that these emitters are from the same manufacturer. The task that discriminates emitters from the same manufacturer is more difficult, because the RF fingerprint features of these emitters are highly similar. However, the overlaps will disappear when more dimensional features are added.

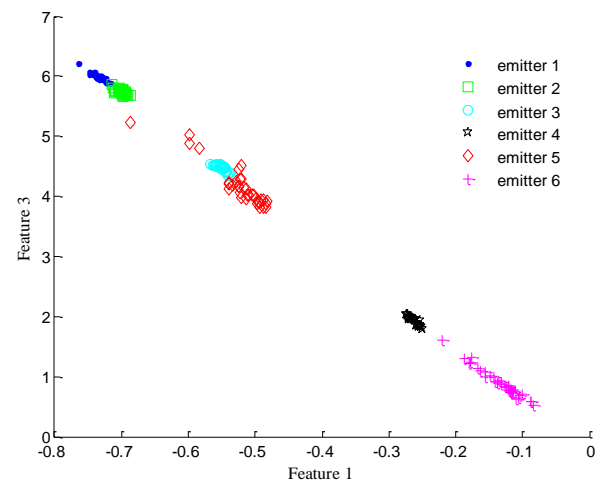

Figure 4. View of the First Feature and the Third Feature

\section{The performance of inter-manufacturer classification}

When we conduct the evaluation of the performance of inter-manufacturer classification, the training set is constructed by selecting data samples from three emitters, one emitter per manufacturer. This will produce eight cases, as illustrated in Table 2. The evaluation is conducted according to the procedure of radiometric identification with GGFA. The one-leave-out strategy is used to train the feature extractor and test the class label of an 'unknown' testing sample. It is shown in Figure 5 that the accuracy of intermanufacturer classification is very high. The correct classification rate (CCR) is almost over $96 \%$ in each case. It can be seen from Figure 5 that the GGFA is useful to discriminate emitters from different manufacturers.

Table 2. Cases of Inter-Manufacturer Classification

\begin{tabular}{ccccccc}
\hline case & Emitter1 & Emitter2 & Emitter3 & Emitter4 & Emitter5 & Emitter6 \\
\hline $\mathrm{a}$ & + & & + & & & + \\
$\mathrm{b}$ & + & & + & + & & \\
$\mathrm{c}$ & + & & & + & + & \\
$\mathrm{d}$ & + & & & & + & + \\
$\mathrm{e}$ & & + & + & + & & \\
$\mathrm{f}$ & & + & + & & & + \\
$\mathrm{g}$ & & + & & + & + & \\
$\mathrm{h}$ & & + & & & + & + \\
\hline
\end{tabular}



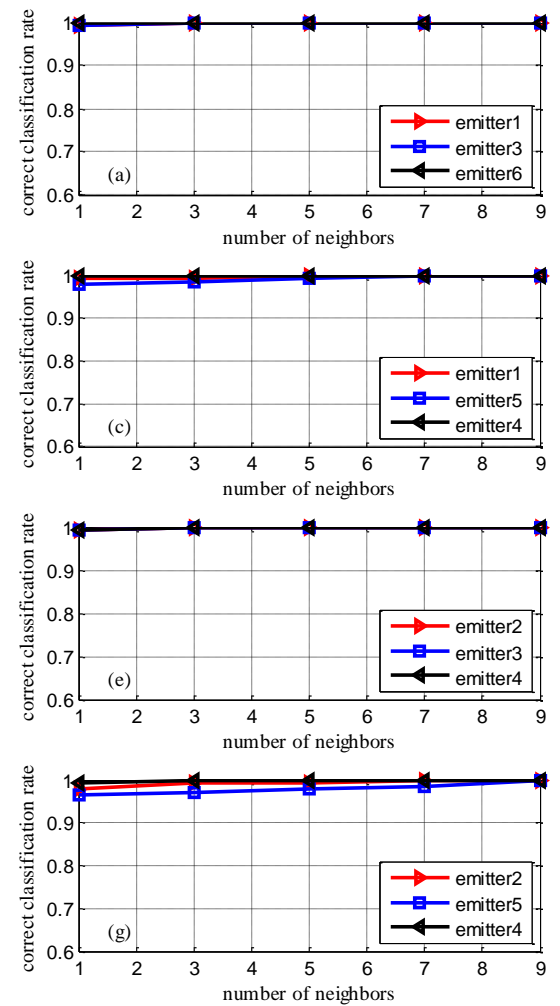
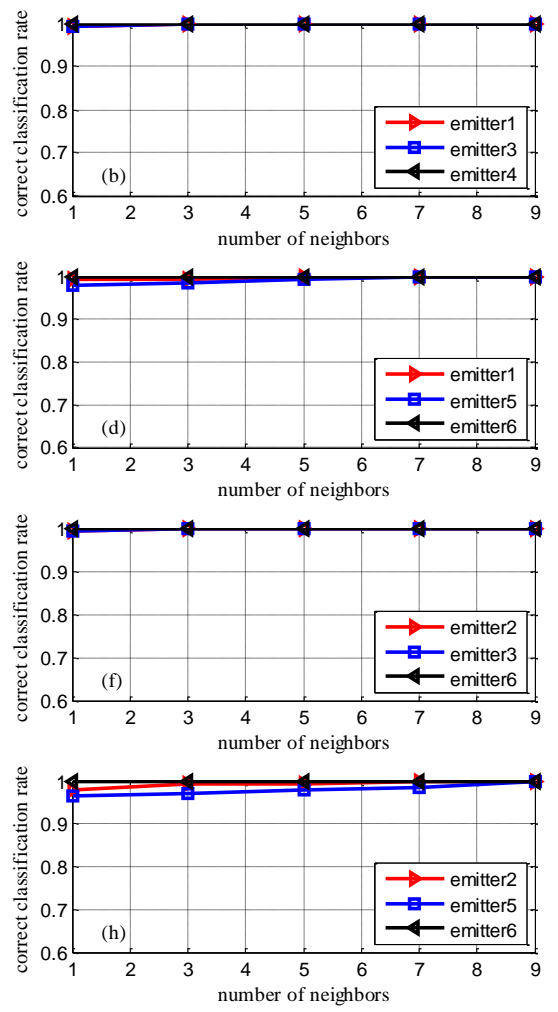

Figure 5. The Accuracy Results with Three Inter-Manufacturer Emitters

\section{The performance of intra-manufacturer classification}

It reveals in the previous subsection that it is difficult to discriminate emitters from the same manufacturer. In order to verify this, we evaluate the classification performance of emitters from the same manufacturer. In this case, data samples are from two emitters of the same manufacturer. The accuracy result of the intra-manufacturer classification is shown in Figure 6. It is noticed that the CCR may be reduced when the similarity of the features of emitters from the same manufacturer is very high. The worst classification performance is achieved, when the emitters are from the second manufacturer (the third emitter and the fifth emitter). The corresponding CCR is only $78 \%$. However, it is shown in Figure 6 that the CCR also may be very high, even if the emitters are from the same manufacturer. For example, the corresponding CCR is over $99 \%$, when the emitters are from the third manufacturer (the fourth emitter and the sixth emitter). It reveals that the proposed method can achieve the best classification accuracy with emitters which have the most unique features, and achieve the worst classification accuracy with emitters which have the most similar features. It can be concluded that the discrimination ability of intra-manufacturer emitters depends on that the emitters are from which manufacturer. 

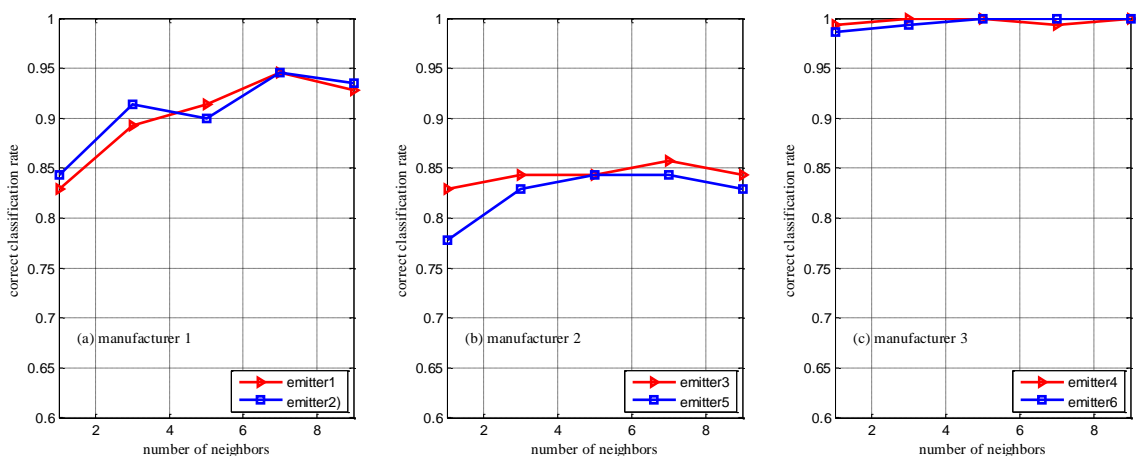

Figure 6. The Accuracy Results with Two Intra-Manufacturer Emitters

\section{E. Parameter discussion}

In this subsection, the effect of selection of parameter $\beta$ (beta) on CCR versus the number of neighbors is investigated. Data samples are from all six emitters in this case and the CCR is calculated as all average correct classification rate. The result is shown in Figure 7. It is noticed that the CCR is reduced when beta varies from 1 to 0 . The underlying reason is that the interclass separability modeled by the penalty graph is more important for the classification task.

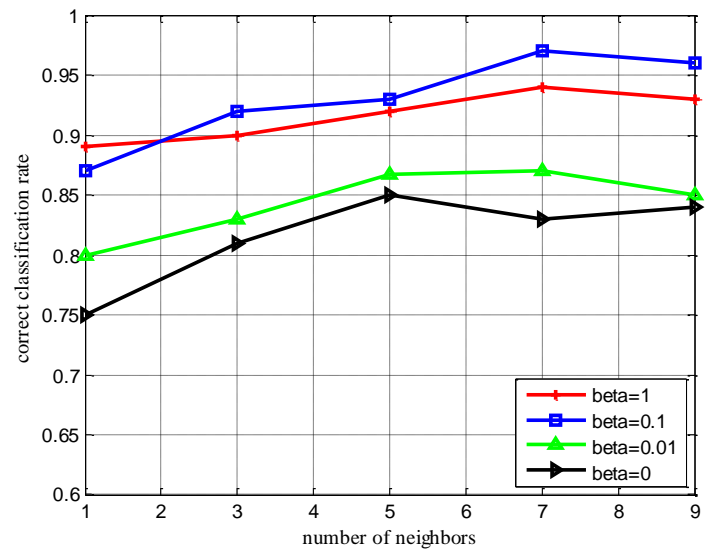

Figure 7. Accuracy Results Considering the Selection of Weight Parameter

\subsection{Comparison of Competitive Methods}

In this subsection, the comparison of the proposed method and other competitive methods is conducted with signals captured in the real-world environment. Burst signals relayed by a satellite transparent transponder from five return channel satellite terminals (RCST) are collected with a signal analyzer. The received satellite signals include the distortions of the satellite transponder and the receiver. However, these distortions are nearly the same for all data samples. The signals are with QPSK modulation and the transmission rate is 5 Mbps. A total of 500 bursts are captured per transmitter and digitized by a 12-bit ADC. The SNR $\left(C / \mathrm{N}_{0}\right)$ is about $30 \mathrm{~dB}$ as measured using spectrometer. The data are preprocessed and added with AWGN. The one-leave-out strategy is also used to conduct the comparison. Data samples from all five emitters are used to construct the training set. The classification is conducted with all methods at each SNR. 


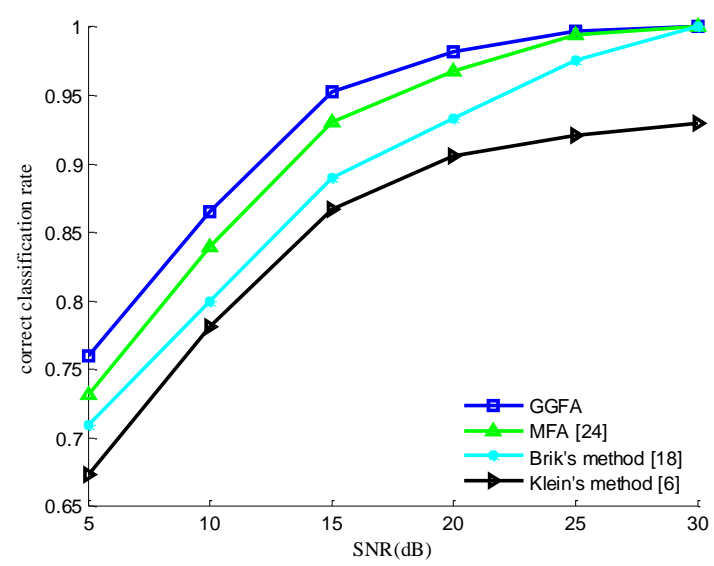

Figure 8. Comparison Results of Different Methods with CCR

Figure 8 shows the comparison results of the proposed method (with $\beta=0.1$ ), Brik's method [18], Klein's method [6], and MFA [24]. It is noticed that the CCRs are over 93\% at $S N R=20 \mathrm{~dB}$, when the evaluations are conducted with the proposed method, MFA and Brik's method. This level of performance is useful for some applications. However, it can be seen from Figure 8 that the proposed method outperforms other methods, when the SNR varies from $5 \mathrm{~dB}$ to $30 \mathrm{~dB}$. Klein's method solely uses the instantaneous amplitude responses, Brik's method concentrates on six modulation error metrics independently, MFA ignores the global information of all samples. The proposed GGFA uses the maximum penalty graph to model the interclass separability which is more important for classification, and uses the minimum intrinsic graph to model the intraclass compactness at the same time.

\section{Conclusion}

We propose a novel radio fingerprint learning algorithm based on characterizing the intraclass compactness and the interclass separability. The interclass separability is modeled by a penalty graph, where not only the connections between the interclass marginal points are calculated, but also the relationships of other interclass vertex pairs are characterized. Meanwhile, the intraclass compactness is modeled by an intrinsic graph, where the relationships of all intraclass vertex pairs are considered. Since both local manifold structure and global discriminant information of the signal data set are considered, the proposed algorithm is suitable for radiometric identification. The extracted features are the discriminative representation of all the factors, which contribute to the minute differences between different radio emitters. From the experiment results on real data sets, we can conclude that the proposed algorithm can discriminate different emitters, and improve the classification accuracy of radiometric identification.

\section{References}

[1] W.M. II. Suski, M.A. Temple, M.J. Mendenhall and R.F. Mills, "RF fingerprinting commercial communication devices to enhance electronic security," Int. J. Electronic Security and Digital Forensics, vol. 1, no. 3, (2008), pp. 301-322.

[2] S.U. Rehman, K.W. Sowerby, C. Coghill, "Analysis of impersonation attacks on systems using RF fingerprinting and low-end receivers," J.Comput. Syst. Sci. vol. 80, no.3, (2014), pp. 591-601.

[3] M. D. Williams, M.A. Temple, D. R. Reising, "Augmenting bit-level network security using physical layer RF-DNA fingerprinting,” IEEE Globecom, Miami, Florida, USA, (2010) Dec 1-6, .

[4] C. K. Dubendorfer, B. W. Ramsey, M.A. Temple, "An RF-DNA verification process for ZigBee networks,” IEEE Int. Conf. MilCom, Orlando, FL, (2012) Nov 1-6. 
[5] B. Danev, S. Kapkun, "Transient-based identification of wireless sensor nodes," IEEE Int. Conf. Information Processing in Sensor Networks, San Francisco, CA, (2009) April 25-36.

[6] R.W. Klein, M.A. Temple, M.J. Mendenhall, D.R. Reising, "Sensitivity Analysis of Burst Detection and RF Fingerprinting Classification Performance,” IEEE Int. Conf. Commun 2009, Dresden, (2009) June 14-18.

[7] O. Ureten, N. Serinken, "Bayesian detection of Wi-Fi transmitter RF fingerprints," Electron. Lett., vol. 41, no.6, (2005), pp. 373-374.

[8] J. Hall, M. Barbeau, E. Kranakis, "Detection of transient in radio frequency fingerprinting using signal phase," Iasted International Multi-Conf. Wireless and Optical Communications, Banff, Canada , (2003) July 13-18.

[9] H.L. Yuan, A.Q. Hu, "Preamble-based detection of Wi-Fi transmitter RF fingerprints," Electron. Lett., vol. 46, no.16, (2010), pp. 1165-1167.

[10] Hall.J, "Detection of rogue devices in wireless network," Carleton University, (2006).

[11] R.W. Klein, M.A. Temple, M.J. Mendenhall, "Application of wavelet based RF fingerprinting to enhance wireless network security,” J.Commun. Networks, vol. 11, no.6, (2009), pp. 544-555.

[12] K. J. Ellis and N. Serinken, "Characteristics of Radio Transmitter Fingerprints," Radio Sci., vol.36, no. 4, (2001), pp. 585-597.

[13] R. M. Gerdes, T. E. Daniels, M. Mina, and S. F. Russell, "Device identification via analog signal fingerprinting: a matched filter approach," Proc. 13th Annual Network and Distrib. Syst. Security Symp., San Diego, CA, (2006).

[14] H. Patel, M. A. Temple, B. W. Ramsey, "Comparison of high-end and low-end receivers for RF-DNA fingerprinting,” IEEE Int. Conf. MilCom, Baltimore, MD, (2014) Oct 24-29.

[15] B. Danev, S. Capkun, "Physical-layer identification of wireless sensor nodes," Eidgenössische Technische Hochschule Zürich, (2008).

[16] P. Scanlon, I. O. Kennedy, and Y. H. Liu, "Feature extraction approaches to RF fingerprinting for device identification in femtocells," Bell Labs Technical Journal, vol.15, no.3, (2010), pp. 141-152.

[17] I. Kennedy, P. Scanlon, F. Mullany, "Radio transmitter fingerprinting: A steady state frequency domain approach," IEEE 68th Vehicular Technology Conference, Singapore, (2008) 1-5.

[18] V. Brik, S. Banerjee, M. Gruteser, and S. Oh, "Wireless device identification with radiometric signatures," 14th ACM international conference on Mobile computing and networking: ACM New York, NY, USA, (2008) 116-127.

[19] D. R. Reising, M.A. Temple, M. J. Mendenhall, "Improved wireless security for gmsk-based devices using RF fingerprints," Int. J. Electronic Security and Digital Forensics, vol. 3, no. 1, (2010), pp. 41-59.

[20] W.C. Suski, M.A. Temple, M.J. Mendenhall, R.F. Mills, "RF fingerprinting commercial communication devices to enhance electronic security," Int. J. Electronic Security and Digital Forensics, vol. 1, no. 3, (2008), pp. 301-322.

[21] A. C. Polak, S. Dolatshahi, D. L. Goeckel, "Identifying wireless users via transmitter imperfections," IEEE Journal on selected areas in communications, vol. 29, no. 7, (2011), pp. 1469-1479.

[22] P. Padilla, J. L. Padilla and J. F. Valenzuela, "Radiofrequency identification of wireless devices based on RF fingerprinting," Electronics Letters, vol. 49, no. 22, (2013), pp.1409-1410.

[23] J. L. Padilla, P. Padilla, and J. F. Valenzuela, "RF fingerprint measurements for the identification of devices in wireless communication networks based on feature reduction and subspace transformation," Measurement, vol. 58, (2014), pp.468-475.

[24] D. Xu, S. Yan, D. Tao, S. Lin and H. Zhang, "Marginal fisher analysis and its variants for human gait recognition and content-based image retrieval," IEEE Trans. Image Processing, vol. 16, no. 11, (2007), pp. 2811-2823.

[25] S. Yan, D. Xu, B. Zhang, H. Zhang, Q. Yang, and S. Lin, "Graph embedding and extensions: A general framework for dimensionality reduction,” IEEE Trans. Pattern Anal. Mach. Intell., vol. 29, no. 1, (2007), pp. $40-51$

\section{Authors}

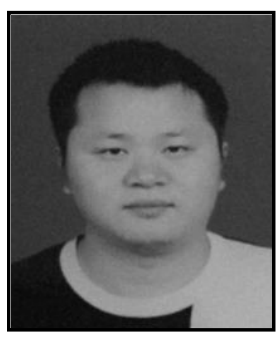

Yongqiang Jia, he received the B.E., M.S. degree from the Information Engineering University, Zhengzhou, in 2003 and 2006. $\mathrm{He}$ is currently a Ph.D candidate in University of Electronic Science and Technology of China. His research interest includes security of wireless communication system. 
International Journal of Signal Processing, Image Processing and Pattern Recognition Vol. 10, No. 4 (2017)

Lu Gan, he received the B.E., M.S. degree and Ph.D. degree in University of Electronic Science and Technology of China (UESTC). He now is a professor in Center for Cyber Security, UESTC. His research interests include Cyber Security, Communication Countermeasure. 